\title{
Collective flow in ultra high energy cosmic rays within CORSIKA
}

\author{
Maowu Nie, ${ }^{a, b, *}$ Hengying Zhang, ${ }^{a, b} \mathbf{L i} \mathbf{Y i}^{a, b}$ Cunfeng Feng ${ }^{a, b}$ and Zhangbu $\mathbf{X u}^{c}$ \\ ${ }^{a}$ Institute of Frontier and Interdisciplinary Science, Shandong University, Qingdao, 266237, China \\ ${ }^{b}$ Key Laboratory of Particle Physics and Particle Irradiation, Ministry of Education, Shandong University, \\ Qingdao, Shandong, 266237, China \\ ${ }^{c}$ Physics Department, Brookhaven National Laboratory, Upton, New York 11976, USA \\ E-mail: li.yi@sdu.edu.cn, fengcf@sdu.edu.cn
}

In heavy ion collisions, the main goal is to create the quark-gluon plasma (QGP) and then study its properties in order to understand quantum chromodynamics at extreme conditions. Collective flow serves as an important probe to study the production and characterize the property of the QGP. In ultra high energy cosmic rays (UHECR), the collision energies can be easily achieved as the current collider experiment. It is naturally to believe the QGP to be created in UHECR collisions. In this work, collective flow are analytically studied within CORSIKA model, with EPOS-LHC for high energy hadronic interaction. The results suggest the collective flow still holds for all charged particles, further study indicates the collective flow of muons are much larger than inclusive charged particles. It seems promising that we can measure the collectivity of muons to search for QGP in UHECR. It can be tested at China's large high altitude air shower observatory (LHAASO) experiments.

$37^{\text {th }}$ International Cosmic Ray Conference (ICRC 2021)

July 12th - 23rd, 2021

Online - Berlin, Germany

\footnotetext{
*Presenter
} 


\section{Introduction}

The most elementary building blocks of matter are leptons and quarks, but quarks (or antiquarks) can not be found isolated in nature, because of quark confinement, one key fundamental phenomena associated with Quantum Chromodynamics (QCD). Namely, quarks and antiquarks are found only confined in hadrons. Another key features of QCD is asymptotic freedom, which states that quarks interact weakly at large energies (or equivalently at short distances). Based on the phenomenon of asymptotic freedom, it was expected that a new state of nuclear matter containing deconfined quarks and gluons, ie QGP, can be produced at high temperatures and energy densities [1].

To search for QGP, a large amount of energy needs to be packed into a limited space volume [2]. Heavy ion collisions (HIC) have been proposed as a more effective way because the initial energy density increases as a power law function with the atomic number while only logarithmically with collision energy. QGP is first observed at Relativistic Heavy Ion Collider (RHIC) [3, 4] at Brookhaven National Laboratory (BNL) and then confirmed at Large Hadron Collider (LHC) at CERN. It is also believed that QGP is also formed in the early universe, a few $\mu s$ after the Big Bang. So the study of QGP can also provide insights on the evolution of our universe.

One of the fundermental mystery in the astronomy field is to find the origin of cosmic rays [5], which are highly energetic ionized nuclei originating from outside the Solar System. Since most of the cosmic rays are charged particles, they will be deflected by the interstellar magnetic field during the propagation process. When they reach the detector, they have already lost the original direction information, so it is impossible to infer the source based on the detected cosmic ray arrival direction. UHECR is less biased the magnetic field and can relect the original direction [6]. When they reach earth, they will collide with a molecule with nuclear interaction in the atmosphere, which creates a shower of particles from the collision point. This shower of particles expands as it travels through the atmosphere through particle decays and additional collisions on atmospheric molecules creating secondary showers.

In UHECR, the energy they carry can cover a wide range, from few $\mathrm{GeV}\left(10^{9} \mathrm{eV}\right)$ up to tens of $\mathrm{EeV}\left(10^{18} \mathrm{eV}\right)$, which can be easily achieved as top RHIC energy (20 ATeV) and top LHC energy (12.5 APeV). As we mentioned above, with such high energy, the QGP is expected to be formed. If it is true, then UHECR and HIC physics are essentially linked, the knowledge we gained from the HIC physics can provide as a baseline for future cosmic rays study.

Experimentally, to signal the production of QGP, a varity of direct and indirect probes are developed, where collecvtive flow is one of the most important direct probes [7]. In non-central heavy ion collisions the initial volume of the interacting system is anisotropic in coordinate space with a almond shape, as shown in Fig. 1. If there is no QGP, the particles are uncorrelated, ie no interactions, the system is a free expansion, the final particle is emitted isotropically in azimuthal angle. However, if the QGP is formed, the QGP expansion will converts the initial state spatial anisotropies into final state momentum anisotropies. These are characterized by Fourier expansion of azimuthal distribution of particle density, $d N / d \phi \propto 1+2 \sum_{n=1}^{\infty} v_{n} \cos n\left(\phi-\Psi_{n}\right)$, where $v_{n}$ and $\Psi_{n}$ represent the amplitude and phase of the $n_{t h}$-order flow vector. $v_{n}$ is under extensive study, since it directly reflect the initial elliptical shape, which is also called elliptic flow.

In this procceding, we investigated the elliptic flow $v_{2}$ in the COsmic Ray SImulations for KAs- 


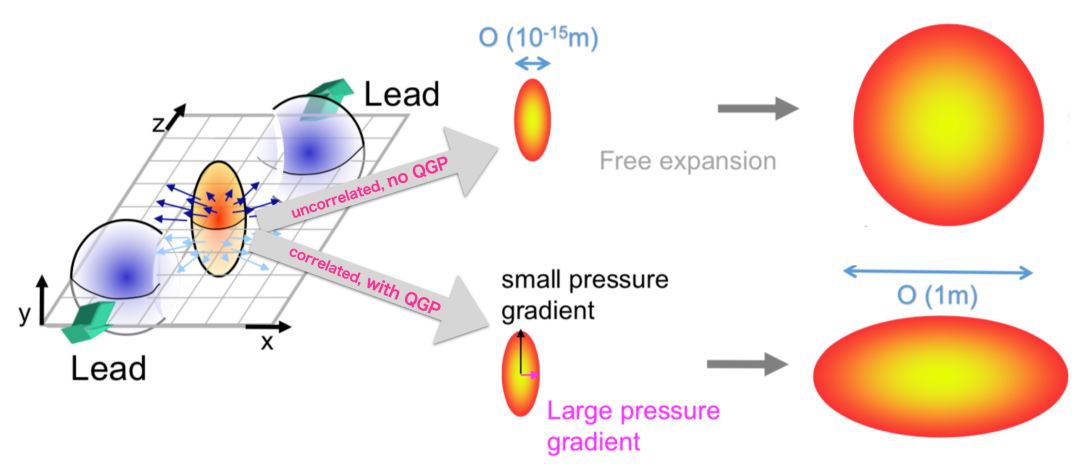

Figure 1: Collective flow: direct probe of QGP

cade(CORSIKA) model with a total enegy $10 \mathrm{TeV}{ }^{4} \mathrm{He}$ as the primary particle. The results suggest the $v_{2}$ still hold for all the final state charge particles. We also notice $\mu v_{2}$ is significantly large than inclusive charge particle $v_{2}$, which may due to $\mu$ suffers less interaction and still holds the anisotropy of the hadron after first collision. It seems like $\mu v_{2}$ can be serve as a choice to search QGP in future cosmic ray measurements.

\section{CORSIKA model}

CORSIKA v76400 is utilized with EPOS-LHC as its high energy model in this study. EPOS is based on the Parton Gribov-Reggie Theory, which is designed to be used for HIC physics, and it includes a parametrized version of collective flow, replicating QGP effect. For simplicity, the primary particle is choosen as vertical Helium $\left({ }^{4} \mathrm{He}\right)$ at $10 \mathrm{TeV}$, only ${ }^{4} \mathrm{He}+{ }^{14} \mathrm{~N}$ collisions are studied, which is equivalent to a $2.5 \mathrm{ATeV}$ fix target collision.

Centrality is a very important variable in heavy ion phsics, it characterizes the area of overlap of the two nuclei during the collision. Centrality is quoted in percentile, from $0-100$, with the $0 \%$ corresponding to the case where the two nuclei overlap completely (or collide head on, referred to as central collisions), while higher values of centrality correspond to cases where the two nuclei overlap partially with increasing separation between their centers. We can get all the hadrons information after the first collision, the centrality is defined with the percentage of the number of hadrons as we commonly defined in HIC.

\section{Results and discussions}

Collective flow study is defined in momentum space, where transverse momentum $p_{T}=$ $\sqrt{p_{x}^{2}+p_{y}^{2}}$, and pseudorapidity $\eta=-\ln \tan \frac{\theta}{2}$, where $\theta$ is the angle between the particle threemomentum $\mathbf{p}$ and the beam axis. However after multiple interactions, the interaction vertex is changed and the beam axis information is smeared via momentum information. To get the first interaction vertex, the pseudorapidity is corrected in the coordinate space as follow: 


$$
\begin{aligned}
r & =\sqrt{x^{2}+y^{2}} \\
\theta & =\operatorname{atan} 2(r, h) \\
\eta & =-\ln \tan \frac{\theta}{2}
\end{aligned}
$$

where, $\mathrm{x}$ and $\mathrm{y}$ are the coordinate space information, $\mathrm{h}$ is the height for the first collision. With this correction, we can get the transverse momentum and pseudorapidity distribution for charge particles, $\mu^{+}$and $\mu^{-}$as shown in Fig. 2. We can find a significant number of charge particles lie at very low $p_{T}$ region $\left(p_{T}<0.2 \mathrm{GeV} / \mathrm{c}\right)$, which means these particles are rest close to 0 in the transver plane and less interact with each other. For the pseudorapidity distribution, we set a kinematic cut with $4<\eta<8$, which is corresponding to a coverage of radius from $10 \mathrm{~m}$ to $1000 \mathrm{~m}$ with respect to the center.
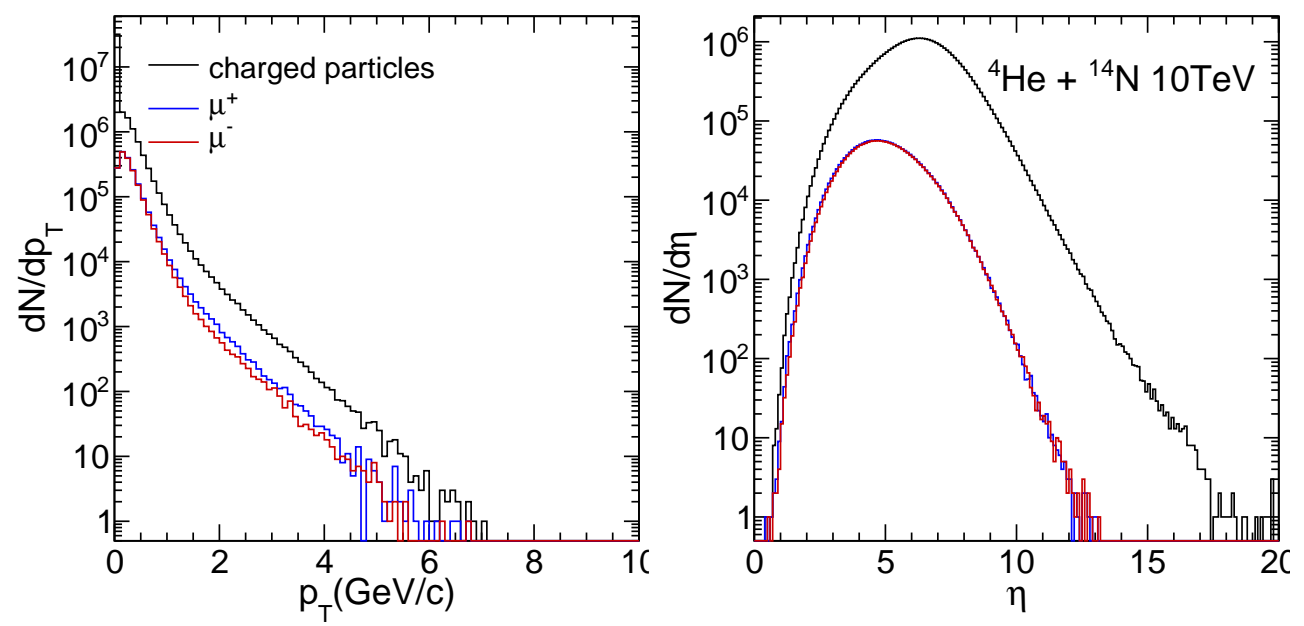

Figure 2: $p_{T}$ and $\eta$ distribution for ${ }^{4} \mathrm{He}+{ }^{14} \mathrm{~N}$ at $10 \mathrm{TeV}$.

The collective flow $v_{2}$ is calculated as a function of $p_{T}$ for charge particles, $\mu^{+}$and $\mu^{-}$at three Centrality intervals. The reaction plane is defined in EPOS model and can be directly extracted event-by-event. We can see $v_{2}$ for charged particles is increasing with $p_{T}$ and then decreasing. It clearly demonstrate a non-zero $v_{2}$ for all the three Centrality intervals. As we known, after the primary cosmic particle has collided with the air molecule, the main part of the first interactions are pions, kaons and baryons. Pions and kaons may decay into other particles, mostly muons. In addition, the secondary particles produce a widespread flash of light in forward direction due to the Cherenkov effect, as well as fluorescence light that is emitted isotropically from the excitation of nitrogen molecules. Thus collectivity may wash out by these process, while muons may still carry as much as the hadron information. $\mu^{+}$and $\mu^{-} v_{2}$ is also shown in Fig. 3 as a function of $p_{T}$ with blue points and red open box, respectively. Muon $v_{2}$ is much larger than the inclusive charged particles, which indicates muons carry more collective information than other particles. The measurements of muon $v_{2}$ is promising to probe QGP in UHECR. 

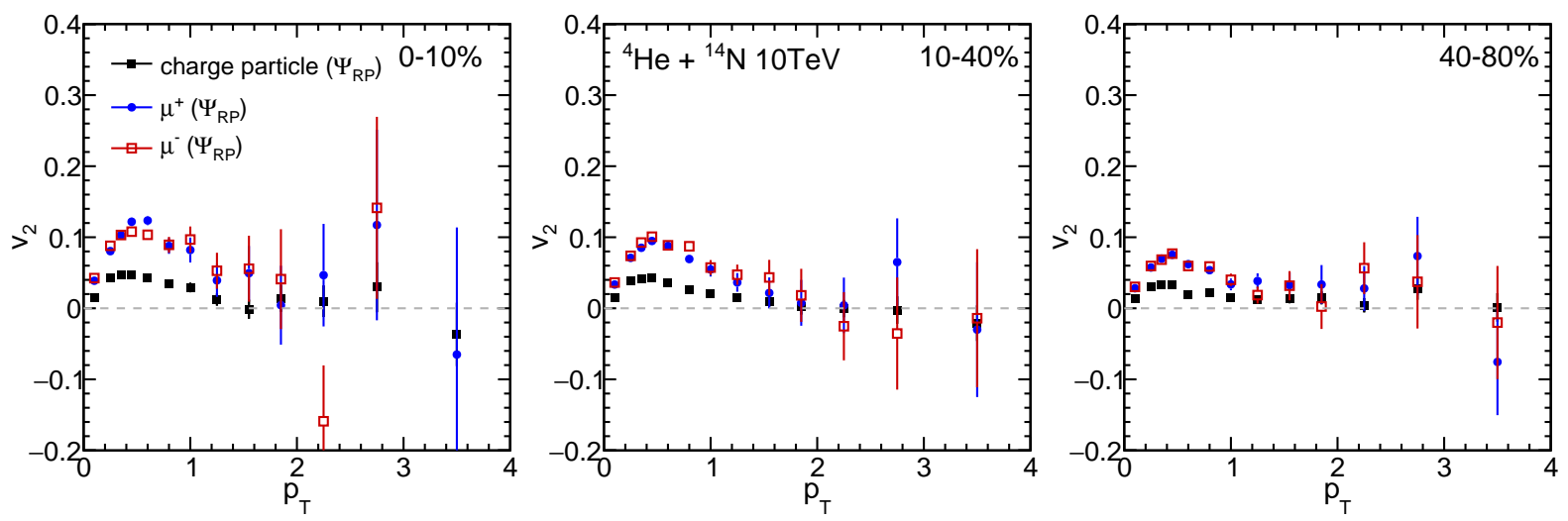

Figure 3: $v_{2}$ as a function of $p_{T}$ at three Centrality bins for ${ }^{4} \mathrm{He}+{ }^{14} \mathrm{~N}$ at $10 \mathrm{TeV}$.

\section{Summary}

Collectivity flow study is studied in UHECR within CORSIKA. ${ }^{4} \mathrm{He}+{ }^{14} \mathrm{~N}$ with a total energy of $10 \mathrm{TeV}$ is utilized in this study. We find the collectivity still holds for final charge particles, and muon $v_{2}$ is a promising approch to capture the collective behavior in UHECR, and it can be tested at LHAASO experiments [8]. Since cosmic rays cover a wider energy ranges and more collision species, fruitful QGP studies are expected in future cosmic ray studies.

\section{References}

[1] E. V. Shuryak, “Theory of Hadronic Plasma," Sov. Phys. JETP 47, 212-219 (1978) IYF-77-34.

[2] T. D. Lee and G. C. Wick, "Vacuum Stability and Vacuum Excitation in a Spin 0 Field Theory," Phys. Rev. D 9, 2291-2316 (1974)

[3] J. Adams et al. [STAR], "Experimental and theoretical challenges in the search for the quark gluon plasma: The STAR Collaboration's critical assessment of the evidence from RHIC collisions," Nucl. Phys. A 757, 102-183 (2005)

[4] K. Adcox et al. [PHENIX], "Formation of dense partonic matter in relativistic nucleus-nucleus collisions at RHIC: Experimental evaluation by the PHENIX collaboration," Nucl. Phys. A 757, 184-283 (2005)

[5] R. Aloiso, E. Coccia and F. Vissani, (Springer, 2018) "Multiple Messengers and Challenges in Astroparticle Physics,"

[6] M. Amenomori, Y. W. Bao, X. J. Bi, D. Chen, T. L. Chen, W. Y. Chen, X. Chen, Y. Chen, Cirennima and S. W. Cui, et al. "First Detection of Photons with Energy Beyond $100 \mathrm{TeV}$ from an Astrophysical Source," Phys. Rev. Lett. 123, no.5, 051101 (2019)

[7] J. Y. Ollitrault, "Anisotropy as a signature of transverse collective flow," Phys. Rev. D 46, 229-245 (1992) 
[8] Z. Cao [LHAASO], "A future project at Tibet: The large high altitude air shower observatory (LHAASO)," Chin. Phys. C 34, 249-252 (2010) 